\title{
Delayed feedback of sampled higher derivatives
}

\author{
Tamas Insperger; Gabor Stepan ${ }^{*}$ Janos Turi ${ }^{\ddagger}$
}

\begin{abstract}
Systems where the present rate of change of state depends on the past values of the higher rates of changes of state are described by so-called advanced functional differential equations (AFDEs). In an AFDE, the highest derivative of the state-space coordinate appears with delayed argument only. The corresponding linearized equations are always unstable with infinitely many unstable poles, and are rarely related to practical applications due to their inherently implicit nature. In this paper, one of the simplest AFDEs, a linear scalar first order system is considered with delayed feedback of the second derivative of the state in the presence of sampling in the feedback loop (i.e., in the case of digital control). It is shown that sampling of the feedback may stabilize the originally infinitely unstable system for certain parameter combinations. The result explains the stable behavior of certain dynamical systems with feedback delay in the highest derivative.
\end{abstract}

Keywords: functional differential equations, advanced argument, sampling, stability, delay.

\section{Introduction}

Functional differential equations (FDEs) are often used to describe hereditary systems in different fields of science and engineering. FDEs can be categorized into retarded, neutral and advanced types (see, e.g., Ėl'sgol'c, 1964, or Kolmanovskii and Myshkis, 1999). If the rate of change of the state depends on the past states of the system, then the corresponding mathematical model is a retarded functional differential equation (RFDE). If the rate of change of the state depends on its own past values as well, then the corresponding equation is called neutral functional differential equation (NFDE). If the rate of change of the state depends on the past values of higher derivatives of the state, then the system is described by an advanced functional differential equation (AFDE). Note that these equations are also referred to as FDEs of retarded, neutral or advanced type.

The reason for the phrase "advanced" can be is demonstrated by the following example. Consider the simple AFDE

$$
\dot{x}(t)=\ddot{x}(t-\tau) .
$$

*Department of Applied Mechanics, Budapest University of Technology and Economics, H-1521 Budapest, Hungary, phone: 36-01-463-1227, fax: 36-01-463-3471, e-mail: inspi@mm.bme.hu

${ }^{\dagger}$ Department of Applied Mechanics, Budapest University of Technology and Economics, H-1521 Budapest, Hungary, phone: 36-01-463-1369, fax: 36-01-463-3471, e-mail: stepan@mm.bme.hu

${ }_{\ddagger}^{\ddagger}$ Programs in Mathematical Sciences, University of Texas at Dallas, Richardson, TX 75083, phone: (972)-883-2183, fax: (972)-883-6622, e-mail: turi@utdallas.edu 
By a $\tau$-shift transformation in time, and by using the new variable $z=\dot{x}$, this equation can be written in the form

$$
\dot{z}(t)=z(t+\tau) .
$$

Here, the rate of change of state is determined by the future values of the state, i.e. an advanced state determines the present rate of change of state.

As opposed to RFDEs and NFDEs, AFDEs are rarely used in practical applications due to their inverted causality explained by (2). Furthermore, the solutions of AFDEs, in general, lose their initial smoothness, and the method of successive integration shows that the solution may not even exist, while the two-sided solutions do exist (Wiener, 1994). Forward solution of AFDEs corresponds to the backward solution of RFDEs that may not exist either. While linear autonomous RFDEs have infinitely many poles on the left half of the complex plane, linear autonomous AFDEs have infinitely many poles on the right half of the complex plane. In this sense, linear autonomous AFDEs are always strongly or infinitely unstable.

The literature of FDEs is quite extensive, we refer the interested reader for example to the texts by Kolmanovskii and Nosov (1986), Stepan (1989), Hale and Lunel (1993), Niculescu (2001), Hu and Wang (2002) or Michiels and Niculescu (2007), just to mention a few. However, these works mostly deal with RFDEs and NFDEs, and AFDEs are not considered since they are said to have no practical significance. Some basic properties of AFDEs together with some special cases are described in the books Èl'sgol'c (1964), Wiener (1994) and Kolmanovskii and Myshkis (1999). Oscillatory conditions for some special AFDEs are discussed in the basic paper by Ladas and Stavroulakis (1982). Shah and Wiener (1982) and Song et al. (2005) analyzed systems with piecewise constant advanced arguments. FDEs with alternately advanced and retarded arguments were analyzed by Filho (1997), by Rodrigues (1997) and by Lv et al. (2007).

In this paper, a linear first order scalar AFDE is considered with piecewise constant argument in the advanced term. The investigation of this system is motivated by an engineering example of a digital control system with the delayed feedback of a derivative higher than that of the present state. The piecewise constant argument corresponds to the sampling effect (zero order hold, $\mathrm{ZOH}$ ) in the digital control. It is shown that the system can be exponentially stable, although the continuous counterpart, the autonomous AFDE with continuous argument in the advanced term, is unstable with infinitely many unstable poles.

\section{Motivation}

The motivation of the current study arose during the analysis of a simple stick balancing problem via feedback control that uses accelerometers to determine the stick's angular position. The mechanical model can be seen in figure 1. The equation of motion reads

$$
J \ddot{\varphi}-H m g \sin \varphi=Q,
$$

where $\varphi$ is the angular position of the pinned stick, $m$ is the mass, $H$ is the distance between the suspension point $O$ and the centre of gravity $C, J$ is the mass moment of inertia with respect to the axis normal to the plane of the figure through point $O$, and $Q$ is the control torque. This model, also called stiffness control, is used to describe self balancing of humans in the sagittal plane (Winter, et al., 1998, Stepan, 2009). 


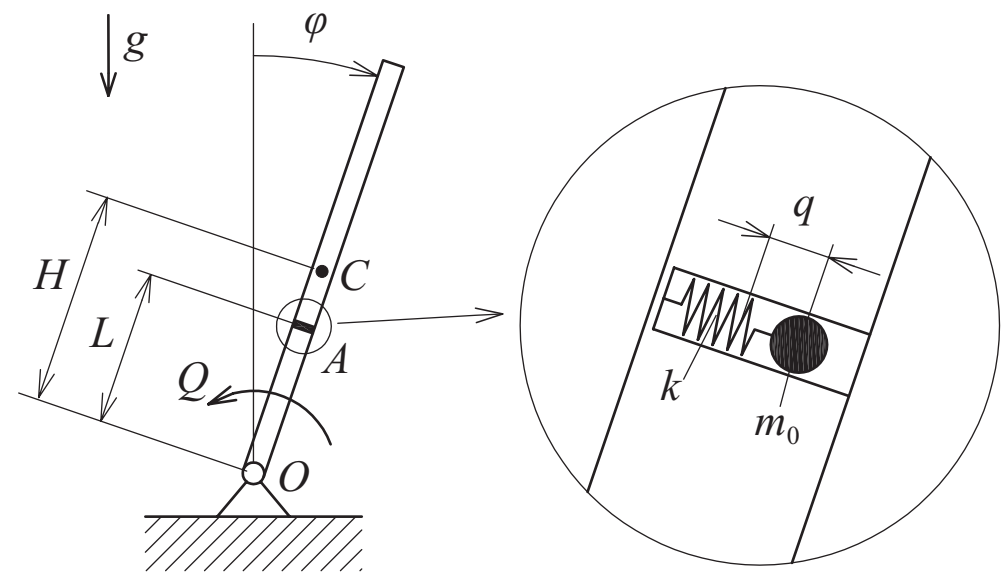

Figure 1: One-DoF model of stick balancing, and the mechanical model of the accelerometer.

In figure 1, the enlarged insert represents the mechanical model of the piezo-accelerometer attached to the stick at point $A$. This accelerometer operates normal to the stick as a mass $m_{0}$ attached to a spring of stiffness $k$ modeling the piezo crystal. The accelerometer's output $y$ is proportional to the displacement $q$ of the mass: $y=K_{A} q$ with $K_{A}[\mathrm{~V} / \mathrm{m}]$ being the characteristic constant of the accelerometer. The displacement $q$ of the mass depends on the angular position $\varphi$ of the rod. If the rod is standing still in an oblique position, then

$$
q=\frac{m_{0} g}{k} \sin \varphi
$$

In an ideal linear case, the output is proportional to the angular position: $y=K \varphi$, where $K=K_{A} m_{0} g / k[\mathrm{~V} / \mathrm{rad}]$. Using the sensor's output $y$ as input in a PD controller with feedback delay $\tau$, the control torque is obtained as

$$
Q=P y(t-\tau)+D \dot{y}(t-\tau)=P K \varphi(t-\tau)+D K \dot{\varphi}(t-\tau),
$$

where $P[\mathrm{~N} / \mathrm{V}]$ and $D[\mathrm{Ns} / \mathrm{V}]$ are the proportional and the differential control gains. In this case, the linearized equation of motion reads

$$
J \ddot{\varphi}(t)-H m g \varphi(t)=P K \varphi(t-\tau)+D K \dot{\varphi}(t-\tau),
$$

This equation is one of the basic examples of RFDEs. The corresponding stability properties are well known and are presented in several textbooks (see, for example, Kolmanovskii and Nosov, 1986, or Stepan, 1989).

If the dynamic effects, namely, the angular acceleration of the rod are also taken into account, then the displacement of the accelerometer reads

$$
q=\frac{m_{0} g}{k} \sin \varphi-\frac{m_{0} L}{k} \ddot{\varphi} .
$$

In this case, the linearized output is

$$
y=K \varphi-K_{1} \ddot{\varphi}
$$

where $K_{1}=K_{A} m_{0} L / k\left[\mathrm{Vs}^{2} / \mathrm{rad}\right]$ and $K$ is the same as above. For this model, a PD controller with feedback delay $\tau$ results in the control torque

$$
\begin{aligned}
Q=P y(t-\tau)+D \dot{y}(t-\tau) & \\
& =P K \varphi(t-\tau)-P K_{1} \ddot{\varphi}(t-\tau)+D K \dot{\varphi}(t-\tau)-D K_{1} \dddot{\varphi}(t-\tau) .
\end{aligned}
$$




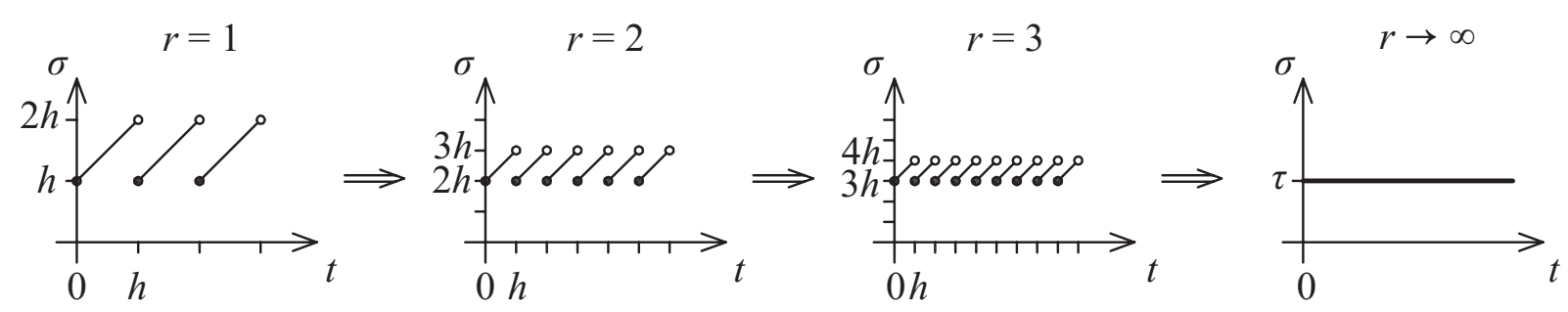

Figure 2: Sampling effect as time-periodic delay.

In this case, the linearized equation of motion reads

$$
J \ddot{\varphi}(t)-H m g \varphi(t)=P K \varphi(t-\tau)-P K_{1} \ddot{\varphi}(t-\tau)+D K \dot{\varphi}(t-\tau)-D K_{1} \dddot{\varphi}(t-\tau) .
$$

This equation is now an AFDE, since the highest (third) derivative appears with a delayed argument only.

In this example, the advanced nature of the system comes from the fact that the accelerometer's signal is affected by the angular acceleration of the rod. Similarly to the previous example, mechanical systems occur that are governed by AFDEs via feeding back the acceleration signal using a PD controller with feedback delay, or by feeding back the jerk in a $\mathrm{P}$ controller with feedback delay. Note that a similar case was investigated by Vyhlídal et al. (2009), where the velocity and the acceleration was fed back proportionally with feedback delay resulting in a NFDE.

A kind of philosophical question is that how such systems can be interpreted in practical applications, when the describing mathematical system is non-causal? Possible answers can be found in the small modeling inaccuracies that are neglected in the model, but they may have important qualitative effects on the behavior of the system. Such a phenomenon is the sampling of digital control, which was not considered in (9) and (10). In digital control, the feedback is held constant for each sampling period $h$ (zero order hold, $\mathrm{ZOH}$ ), i.e., the control torque reads

$$
\begin{aligned}
Q=P y\left(t_{j}-r h\right)+D \dot{y}\left(t_{j}-r h\right) & =P K \varphi\left(t_{j}-r h\right)-P K_{1} \ddot{\varphi}\left(t_{j}-r h\right) \\
& +D K \dot{\varphi}\left(t_{j}-r h\right)-D K_{1} \dddot{\varphi}\left(t_{j}-r h\right), \quad t \in\left[t_{j}, t_{j+1}\right),
\end{aligned}
$$

where $t_{j}=j h, j \in \mathbb{Z}$ and $h$ is the sampling period such that $\tau=r h$ with $r$ being an integer. Note that the sampled output can also be represented in the form

$$
y\left(t_{j}-r h\right)=y(t-\sigma(t)), \quad t \in\left[t_{j}, t_{j+1}\right)
$$

where

$$
\sigma(t)=\tau-t_{j}+t, \quad t \in\left[t_{j}, t_{j+1}\right) .
$$

is a time-periodic time delay shown in figure 2. In fact, the sampling effect introduces a periodic parametric excitation at the time delay according to (13). It can be seen that the time-periodic time delay $\sigma(t)$ tends to the constant delay $\tau$ as the sampling period $h$ tends to 0 .

Note that the sampling effect presented above is equivalent to the zeroth order semidiscretization method of RFDEs (see Insperger et al., 2008). Semi-discretization is a discretization technique, where only the terms with delayed arguments are discretized while the other non-delayed terms are left in their original form. It is known that discretization 

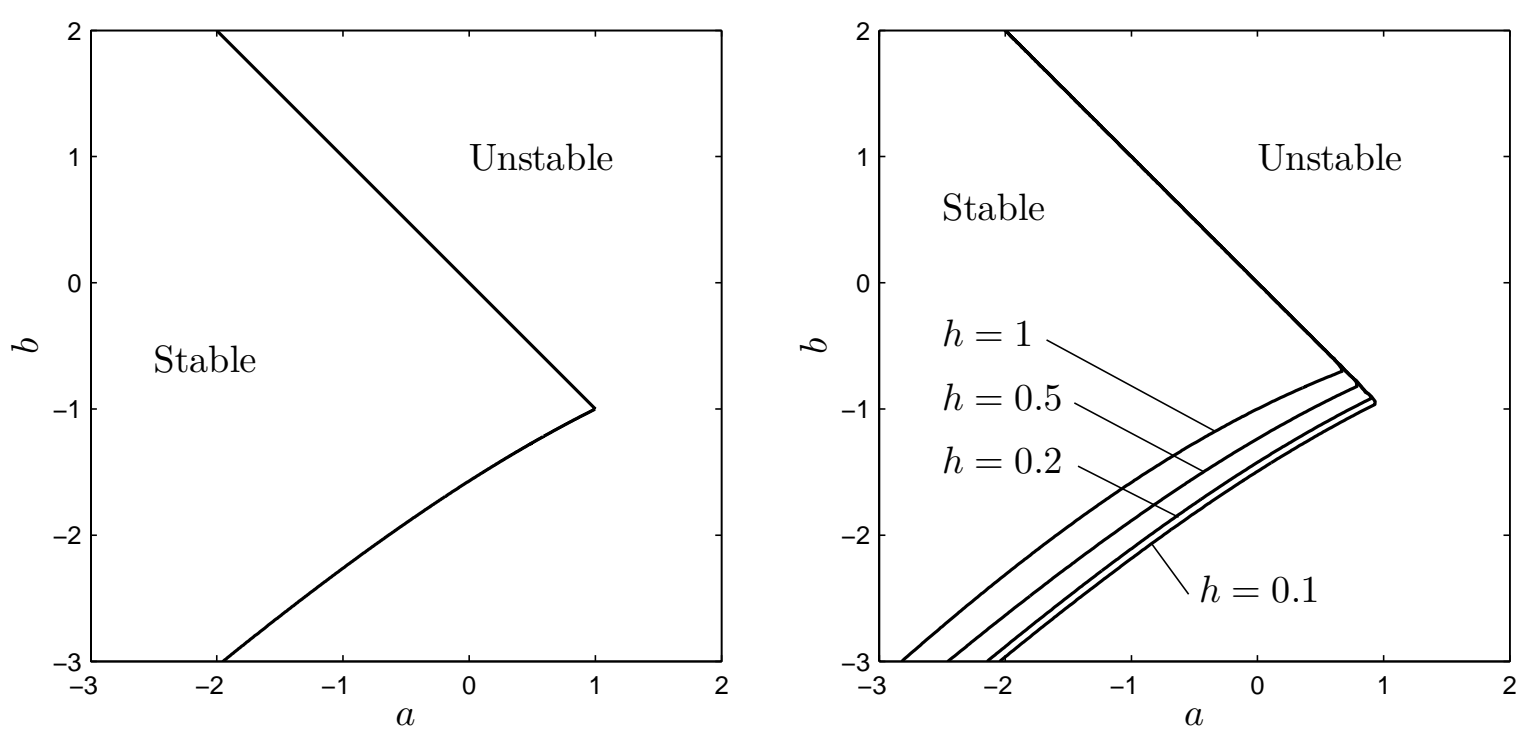

Figure 3: Left: stability chart for the RFDE (14) for $\tau=1$. Right: stability charts for the sampled RFDE (15) for $\tau=1$ and for different sampling periods $h$.

techniques preserve exponential stability for RFDEs (Győri et al., 1998), however, for NFDEs, this is not true in general, as it was shown by Fabiano and Turi (1999). Preservation of exponential stability of NFDEs under delay perturbations was also analyzed by Györi and Hartung (2001). Here, it will be shown for AFDEs that the discretized (or sampled) system can be stable in spite of the fact that the original AFDE is unstable with infinitely many unstable poles.

\section{Delayed, neutral and advanced FDEs}

In this section, first order FDEs of delayed, neutral and advanced type are considered with and without sampling effect in the delayed term. Stability properties with respect to the system parameters are analyzed.

\subsection{First order delay differential equation}

Consider the first order delay differential equation

$$
\dot{x}(t)=a x(t)+b x(t-\tau) .
$$

This equation is a special type of Cushing's equation (Cushing, 1977) and it is often referred to as one of the simplest basic examples for a delayed system (see, e.g., Hale and Lunel, 1993, Stepan, 1989 or Michiels et al., 2002). The stability condition for the parameters $a$ and $b$ was first presented by Hayes (1950). The corresponding stability chart can be seen in the left panel of figure 3 .

Equation (14) can also be considered as a control system with delayed feedback of the state, where $a$ is the system parameter, $b$ is the control gain and $\tau$ is the feedback delay. In case of digital control, zero order hold arises in the feedback loop, thus, the argument 
of the delayed term becomes piecewise constant. The corresponding equation reads

$$
\dot{x}(t)=a x(t)+b x\left(t_{j}-r h\right), \quad t \in\left[t_{j}, t_{j+1}\right),
$$

where $h$ is the sampling period, $t_{j}=j h$ and $j, r \in \mathbb{Z}$ such that $r h=\tau$. Due to the piecewise constant argument of the delayed term, (15) can be solved over each sampling period $\left[t_{j}, t_{j+1}\right)$, and the finite dimensional discrete map can be constructed

$$
y_{j+1}=\Phi y_{j}
$$

where

$$
y_{j}=\left(\begin{array}{c}
x\left(t_{j}\right) \\
x\left(t_{j-1}\right) \\
x\left(t_{j-2}\right) \\
\vdots \\
x\left(t_{j-r}\right)
\end{array}\right) \quad \text { and } \Phi=\left(\begin{array}{ccccc}
\mathrm{e}^{a h} & 0 & \ldots & 0 & \frac{b}{a}\left(\mathrm{e}^{a h}-1\right) \\
1 & 0 & \ldots & 0 & 0 \\
0 & 1 & \ldots & 0 & 0 \\
\vdots & & \ddots & & \vdots \\
0 & 0 & \ldots & 1 & 0
\end{array}\right) .
$$

The system is asymptotically stable if the eigenvalues of the monodromy matrix $\Phi$ are in modulus less than one. The corresponding stability charts can be seen in the right panel of figure 3 for $\tau=1$ and for different sampling periods. It can be seen that by decreasing the sampling period $h$ while keeping $r h=\tau=1$ constant, the stability boundaries converge to that of (14). This is due to the fact that equation (15) is the zeroth order semi-discretization of equation (14) according to Insperger et al. (2008). Since semi-discretization preserves asymptotic stability of the original equation (14) (see Hartung et al., 2006), it can be used to obtain approximations of the stability charts, as it is demonstrated in figure 3 .

\subsection{First order differential equation of neutral type}

Consider the first order differential equation of neutral type

$$
\dot{x}(t)=a x(t)+b \dot{x}(t-\tau) .
$$

This equation can be considered as a control system with delayed feedback of the state's derivative. The condition for asymptotic stability is $a<0$ and $|b|<1$ (see Kolmanovskii and Nosov, 1986). The corresponding stability chart can be seen in the left panel of figure 4 .

The sampled counterpart of equation (18) reads

$$
\dot{x}(t)=a x(t)+b \dot{x}\left(t_{j}-r h\right), \quad t \in\left[t_{j}, t_{j+1}\right) .
$$

Similarly to equation (15), equation (19) can be transformed into a discrete map of the form

$$
z_{j+1}=\Psi z_{j}
$$

where

$$
z_{j}=\left(\begin{array}{c}
x\left(t_{j}\right) \\
\dot{x}\left(t_{j}\right) \\
\dot{x}\left(t_{j-1}\right) \\
\vdots \\
\dot{x}\left(t_{j-r}\right)
\end{array}\right) \quad \text { and } \Psi=\left(\begin{array}{ccccc}
\mathrm{e}^{a h} & 0 & \ldots & 0 & \frac{b}{a}\left(\mathrm{e}^{a h}-1\right) \\
a \mathrm{e}^{a h} & 0 & \ldots & 0 & b \mathrm{e}^{a h} \\
0 & 1 & \ldots & 0 & 0 \\
\vdots & & \ddots & & \vdots \\
0 & 0 & \ldots & 1 & 0
\end{array}\right)
$$



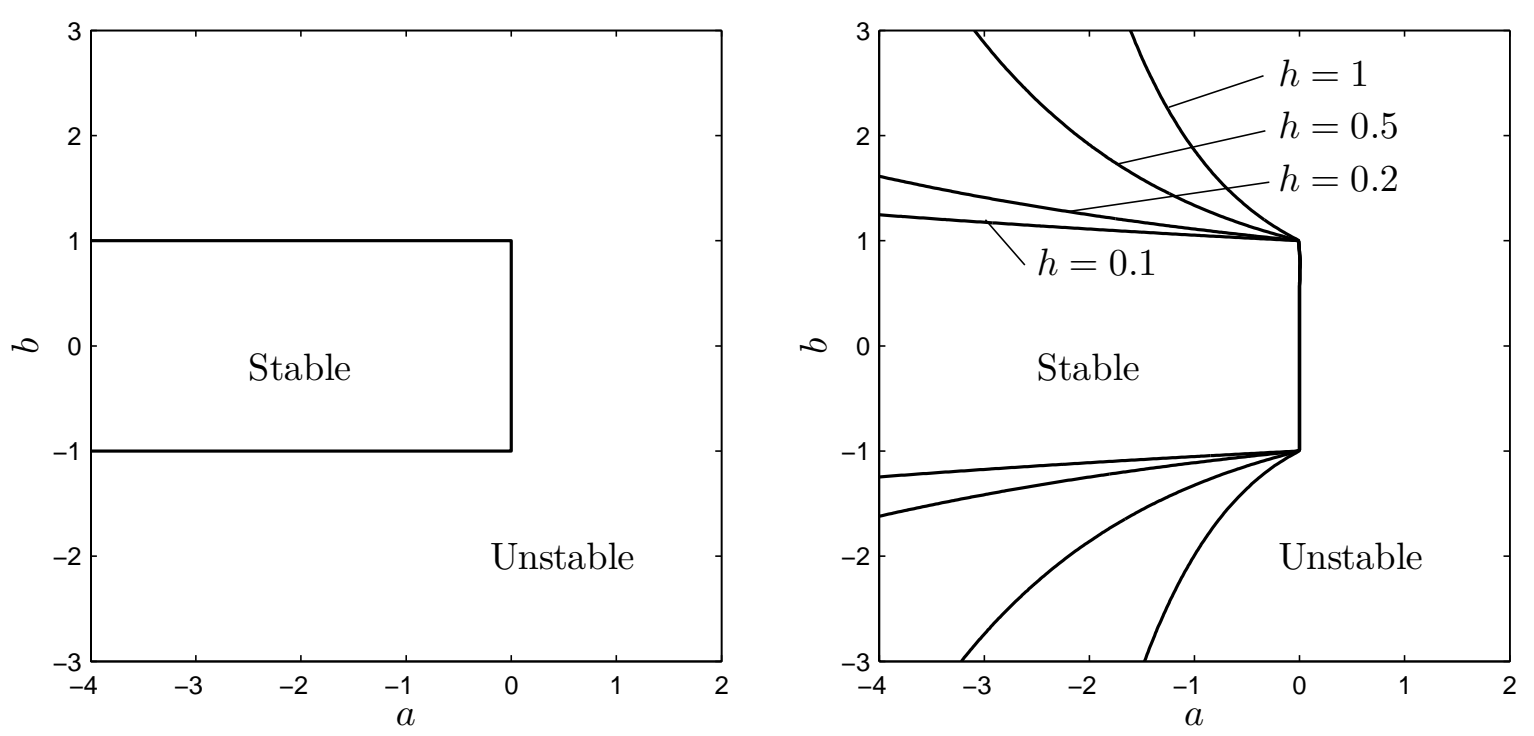

Figure 4: Left: stability chart for the NFDE (18) for $\tau=1$. Right: stability charts for the sampled NFDE (19) for $\tau=1$ and for different sampling periods $h$.

The system is asymptotically stable if the eigenvalues of the monodromy matrix $\Psi$ are in modulus less than one. The corresponding stability charts can be seen in the right panel of figure 4 for $\tau=1$ and for different sampling periods. The stability boundaries of the sampled system (19) seem to converge to that of the original (non-sampled) one (19). Note that, although the stability charts of the sampled system in figure 4 seem to converge to the chart of equation (18), the above discretization does not necessarily preserves exponential stability for NFDEs generally (Fabiano and Turi, 1999).

\subsection{First order differential equation of advanced type}

Consider the first order differential equation of advanced type

$$
\dot{x}(t)=a x(t)+b \ddot{x}(t-\tau) .
$$

This system is asymptotically stable for $b=0$ and $a<0$, and is unstable for any other parameter combinations (see, for example, Ėl'sgol'c, 1964). If $b \neq 0$, then the system has infinitely many unstable poles (i.e., roots of the characteristic equation with positive real parts).

The sampled counterpart of equation (22) reads

$$
\dot{x}(t)=a x(t)+b \ddot{x}\left(t_{j}-r h\right), \quad t \in\left[t_{j}, t_{j+1}\right) .
$$

This is an equation of advanced type, again, however, the argument of the advanced term is piecewise constant. Another form of equation (23) is

$$
\dot{x}(t)=a x(t)+b \ddot{x}(t-\sigma(t)),
$$

where $\sigma(t)$ is a time-periodic time delay defined in (13) and presented in figure 2. Similarly to the previous RFDE and NFDE cases, a discrete map can be constructed by step-by-step solution in the form

$$
w_{j+1}=\Theta w_{j}
$$



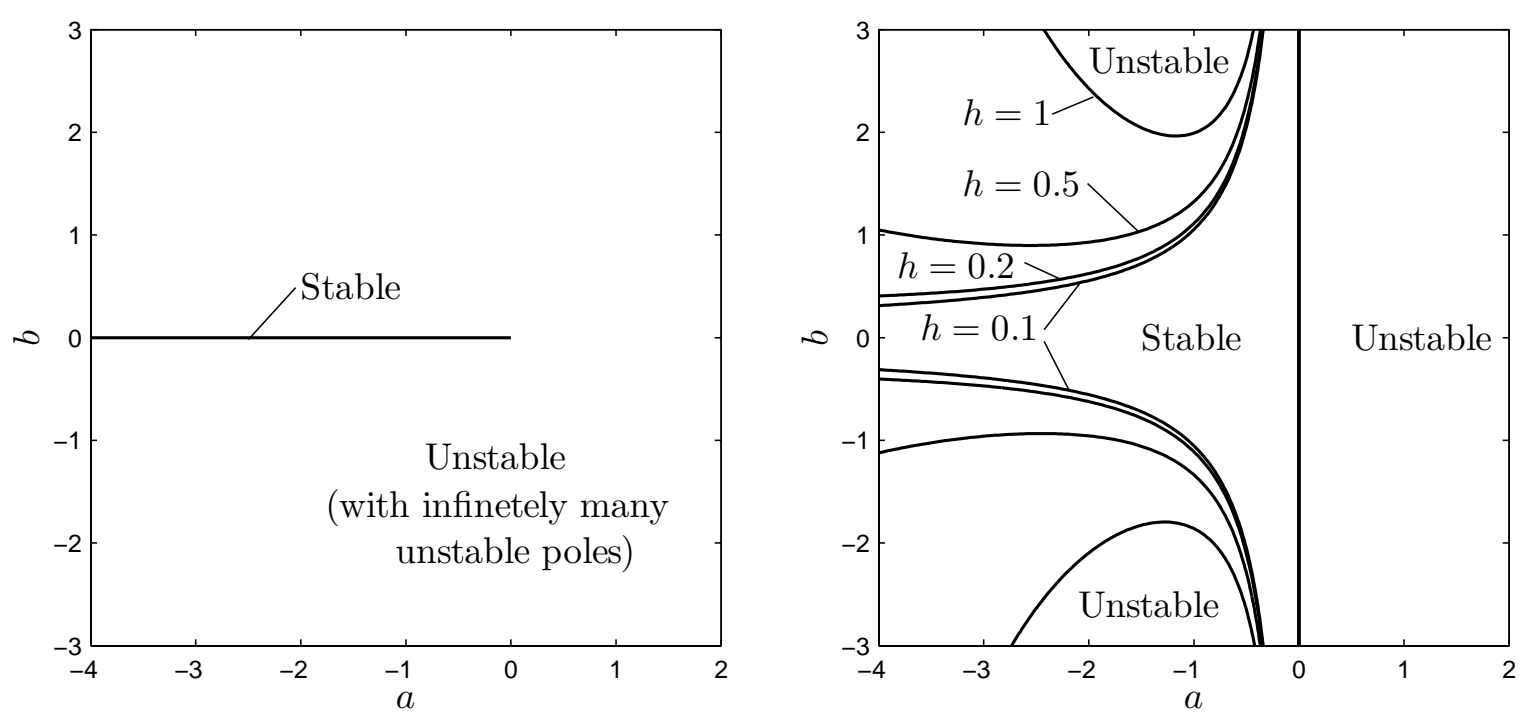

Figure 5: Left: stability chart for the AFDE (22) for $\tau=1$. Right: stability charts for the sampled AFDE (23) for $\tau=1$ and for different sampling periods $h$.

where

$$
w_{j}=\left(\begin{array}{c}
x\left(t_{j}\right) \\
\ddot{x}\left(t_{j}\right) \\
\ddot{x}\left(t_{j-1}\right) \\
\vdots \\
\ddot{x}\left(t_{j-r}\right)
\end{array}\right) \quad \text { and } \quad \Theta=\left(\begin{array}{ccccc}
\mathrm{e}^{a h} & 0 & \ldots & 0 & \frac{b}{a}\left(\mathrm{e}^{a h}-1\right) \\
a^{2} \mathrm{e}^{a h} & 0 & \ldots & 0 & a b \mathrm{e}^{a h} \\
0 & 1 & \ldots & 0 & 0 \\
\vdots & & \ddots & & \vdots \\
0 & 0 & \ldots & 1 & 0
\end{array}\right) .
$$

Again, this system is asymptotically stable if the eigenvalues of the monodromy matrix $\Theta$ are in modulus less than one. The corresponding stability charts can be seen in the right panel of figure 5 for $\tau=1$ and for different sampling periods. It can be seen that while the original equation (22) is always unstable for $b \neq 0$ with infinitely many unstable roots, the sampled system can be stable even for $b \neq 0$. Furthermore, it can be seen that the stability boundaries do not converge to the boundaries of the non-sampled system.

\section{AFDE stability diagrams}

In order to obtain an insight to the stability properties of equation (23), the eigenvalues of the corresponding monodromy matrix $\Theta$ should be analyzed in detail. For a given sampling period $h$, the matrix $\Theta$ has $r+2=\tau / h+2$ eigenvalues that can be computed numerically. The magnitude $\left|\mu_{1}\right|$ of the critical (maximum in modulus) eigenvalue $\mu_{1}$ characterizes the decay of the system's oscillations for perturbations, i.e., $\left\|w_{j+1}\right\| \leq\left|\mu_{1}\right|\left\|w_{j}\right\|$. Figure 6 shows the contour plots of the maximum eigenvalues for different sampling periods. Stability boundaries are denoted by the contour lines where $\left|\mu_{1}\right|=1$ (these are denoted by thick lines). It can be seen that the stable domains in the stability diagrams do not disappear as the sampling period is decreasing, however, the magnitude of the critical eigenvalue within the domain of stability approaches 1 (see the contour lines and the associated modulus numbers). 

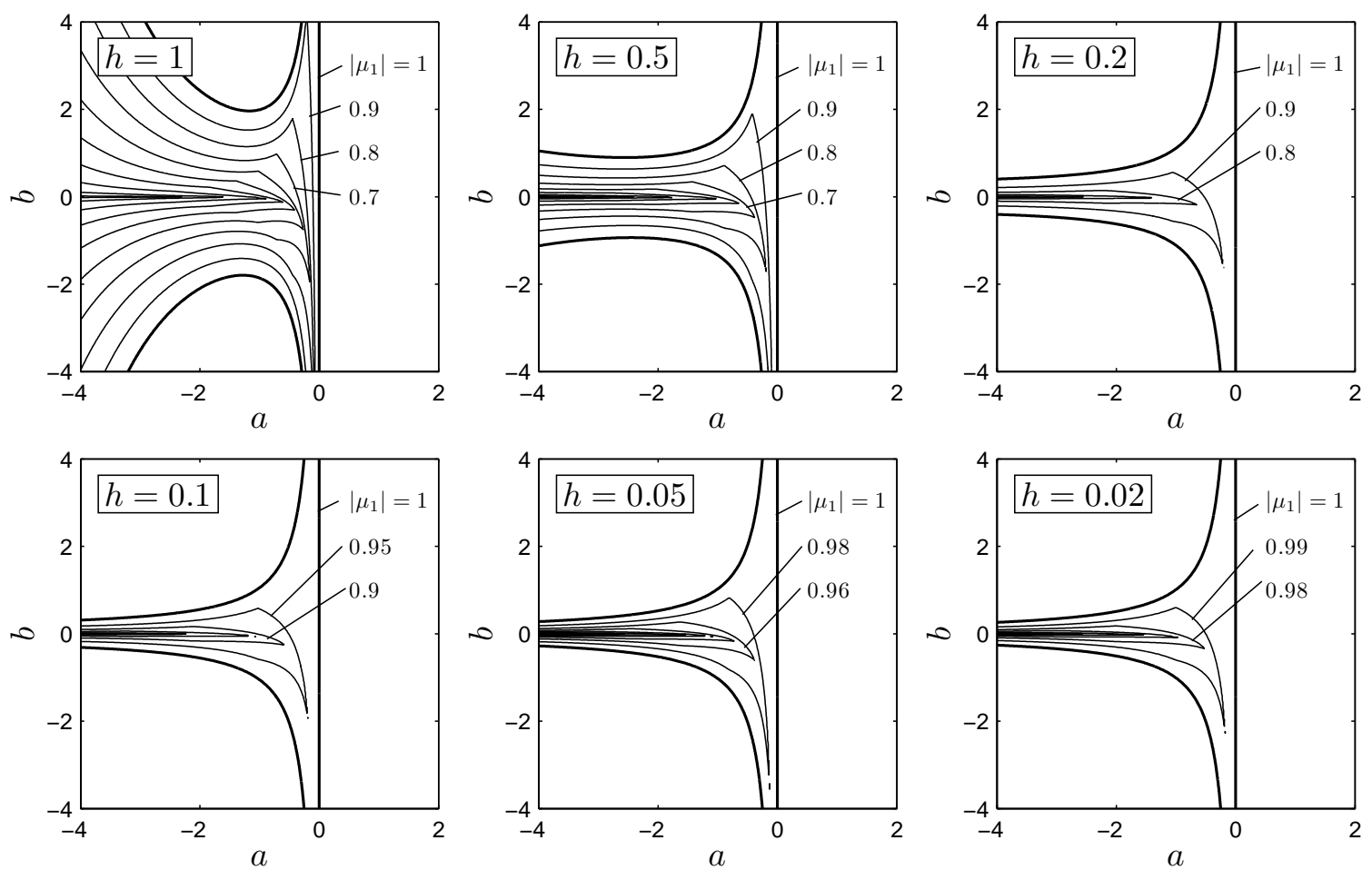

Figure 6: Contour plots for the critical eigenvalues of the sampled AFDE (23) for $\tau=1$ and for different sampling periods $h$.

Figure 7 shows the number of unstable poles for system (23). The stable domains are denoted by 0 . It can be seen that as the sampling period $h$ is decreasing, i.e., as the size of the monodromy matrix is increasing, the number of unstable poles is increasing in the unstable domains. The number of unstable poles are in the range of $\tau / h=r$.

\section{$5 \quad$ Numerical analysis of the transition $h \rightarrow 0$}

In order to analyze the transition between the sampled AFDE (23) and the original AFDE (22), stability diagrams in the plane of $h$ and $b$ are constructed for fixed negative $a$. These diagrams can be determined numerically by increasing the parameter $b$ stepby-step until the magnitude of the critical eigenvalue gets larger than 1 . This gives the stability boundaries for positive $b$. Similarly, the stability boundaries for negative $b$ are obtained by decreasing $b$ step-by-step. Figure 8 presents these diagrams for $a=-1,-2$, -5 and -10 . These numerical results show that the stability domain does not disappear as the sampling period $h$ tends to zero. Furthermore, they suggest that the critical value of $b$ converges to $\pm 1 / a$. This latter feature can easily be confirmed by writing the characteristic equation of the monodromy matrix $\Theta$ in the form

$$
\left(\mu-\mathrm{e}^{a h}\right)\left(\mu^{r+1}-a b \mathrm{e}^{a h}\right)=a b \mathrm{e}^{a h}\left(\mathrm{e}^{a h}-1\right) .
$$

For small sampling period $h$, the roots can be approximated as

$$
\mu_{1} \approx 1+a h \quad \text { and } \quad \mu_{l} \approx(a b(1+a h))^{\frac{1}{r+1}} \mathrm{e}^{\mathrm{i} \frac{2 l \pi}{r+1}}, \quad l=2,3, \ldots, r+2 .
$$

The condition for asymptotic stability in this case is $a<0$ with $|a b(1+a h)|<1$. This confirms that as $h$ tends to zero, the stability boundaries in the plane $(a, b)$ tend to 

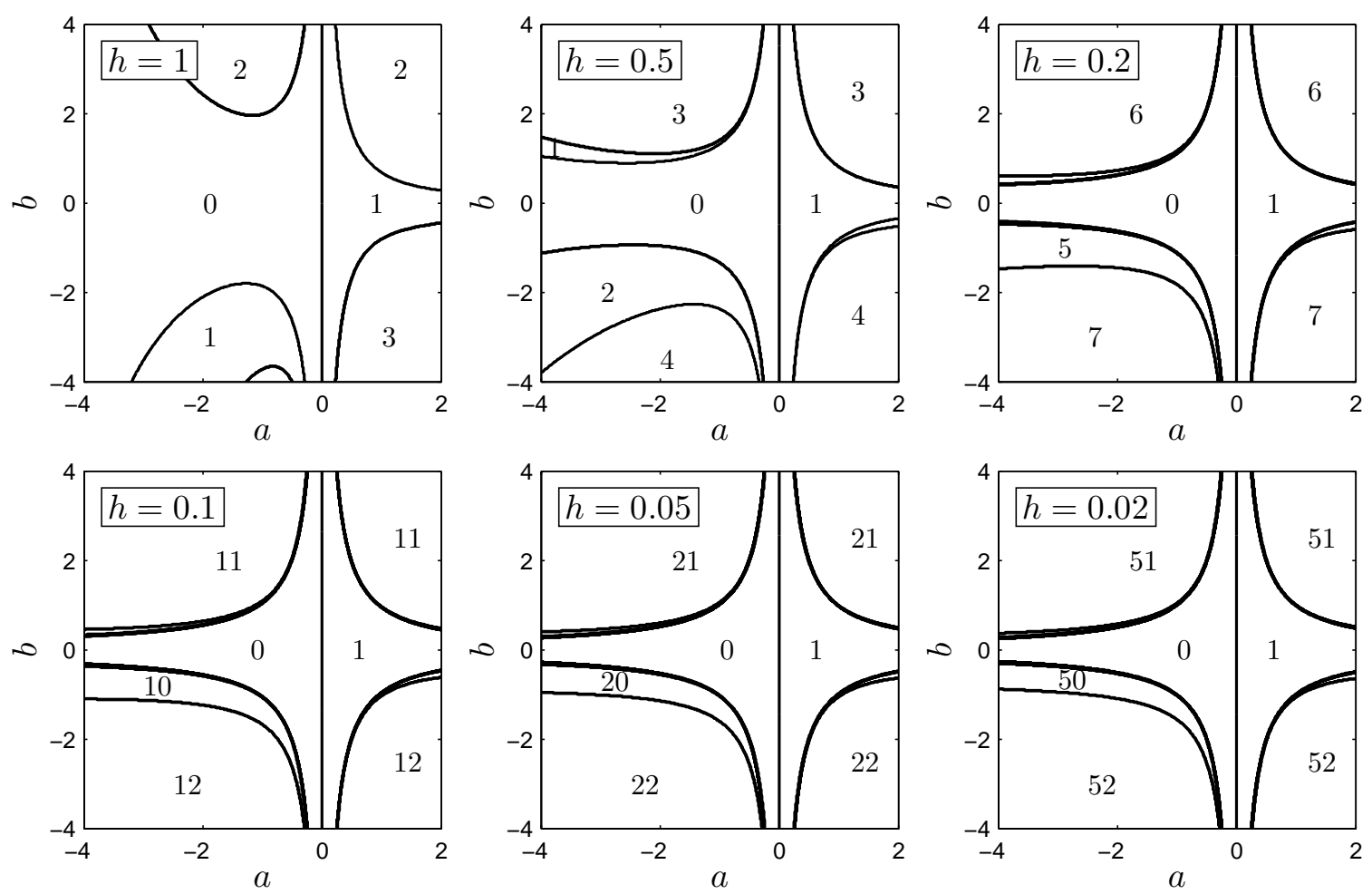

Figure 7: Number of unstable eigenvalues of the sampled AFDE (23) for $\tau=1$ and for different sampling periods $h$.

$b= \pm 1 / a$ with $a<0$. On the other hand, the transition $h \rightarrow 0$ corresponds to the original AFDE, i.e., in this case the system has infinitely many unstable poles if $h=0$ and $b \neq 0$. This shows that as $h \rightarrow 0$, the system is stable if $|b|<-1 / a$ with $a<0$, but in the limit case, i.e., when $h=0$, "suddenly", infinitely many poles become unstable. This phenomenon is presented in figure 9 .

The approximations in equation (28) show that as the sampling period $h$ gets smaller and smaller, one pole $\left(\mu_{1}\right)$ approaches to 1 , while the other $(r+1)$ poles cluster round the circle of radius $a b$ in the complex plane. This phenomenon is presented in figure 10 for some special parameter combinations. Three special cases are considered:

- $a=-1, b=2.5$ : The system is unstable for any sampling periods $h$. The number of unstable poles is increasing as $h$ is decreasing. For $h<0.2$, the magnitude of the critical eigenvalue is decreasing, the poles approaches the unit circle from outside.

- $a=-1, b=1.5$ : The system is stable for $h=1$ but unstable for any other $h<1$. The number of unstable poles is increasing as $h$ is decreasing. The magnitude of the critical eigenvalue is decreasing, the poles approaches the unit circle from outside.

- $a=-1, b=0.5$ : The system is stable for all $h>0$. The number of stable poles is increasing as $h$ is decreasing. The magnitude of the critical eigenvalue is increasing, the poles approaches the unit circle from inside.

The magnitude $\left|\mu_{1}\right|$ of the critical eigenvalue is given for each plots. These diagrams demonstrate how the jump of infinitely many poles outside the unit circle appears as $h \rightarrow 0$. 

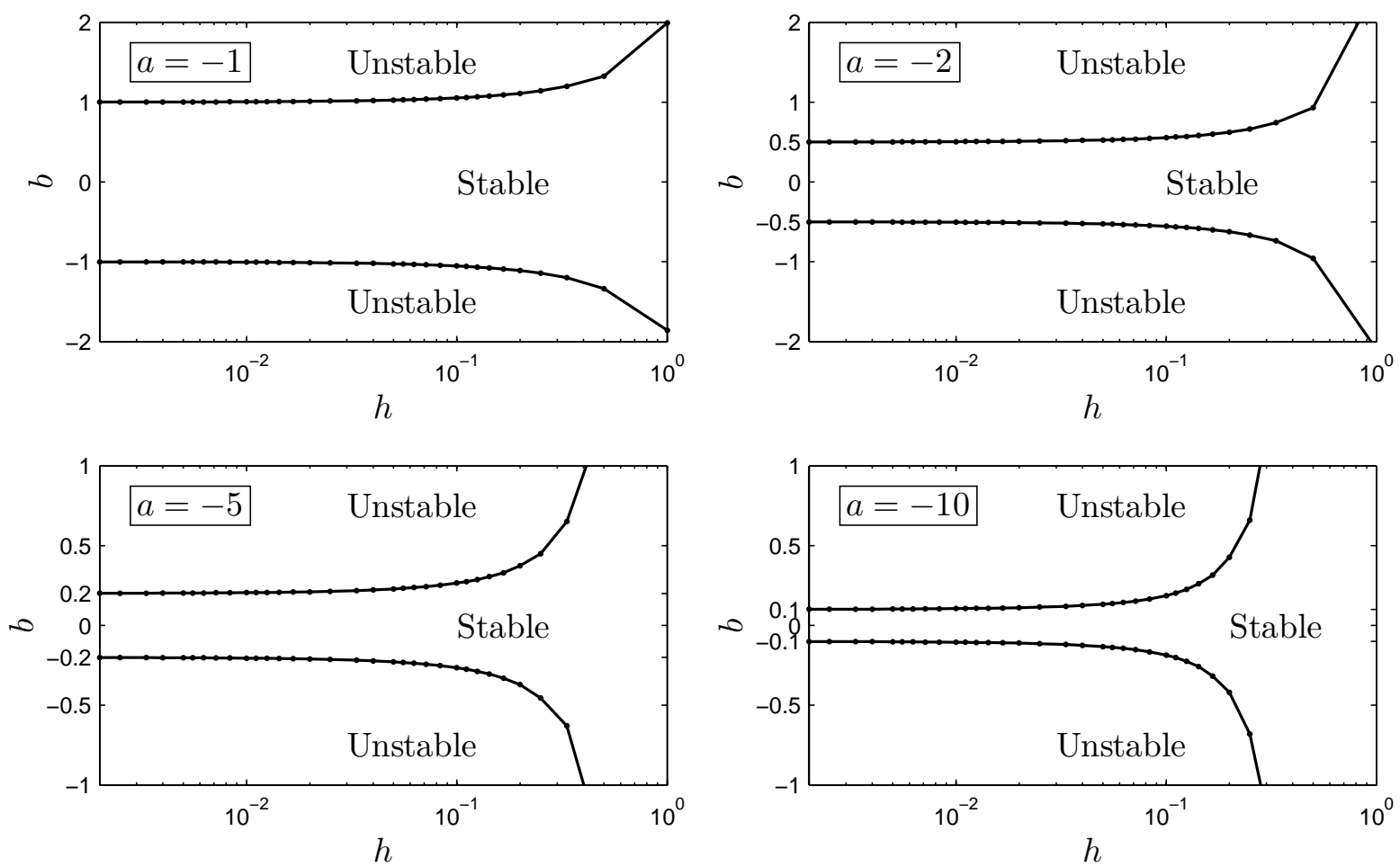

Figure 8: Stability domains for the sampled AFDE (23) in the plane $(h, b)$ for $\tau=1$ and for different system parameters $a$.

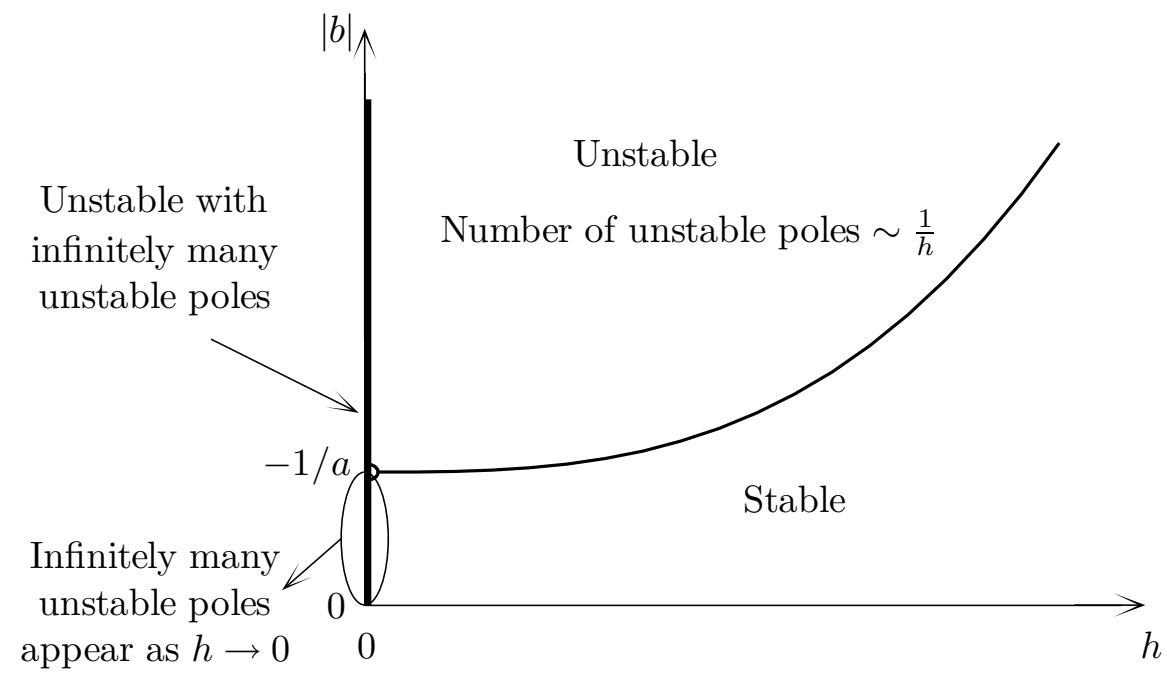

Figure 9: Sketch of the stability properties of the sampled AFDE $(23)$ in the plane $(h, b)$ for fixed $a<0$. 

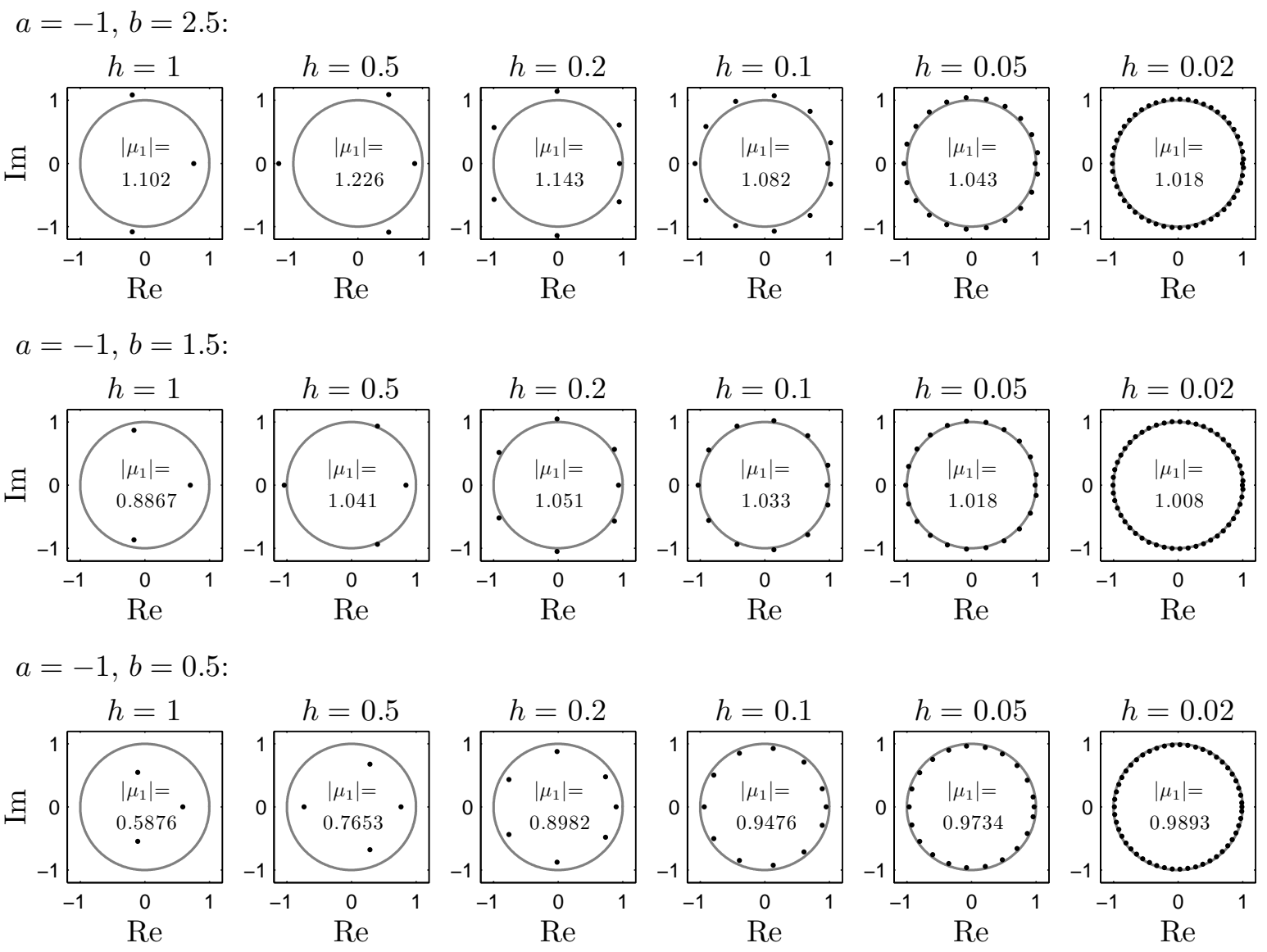

Figure 10: Location of the poles for different parameter combinations. The unit circle is denoted by grey line.

\section{Conclusion}

In this paper, the AFDE (22) and its sampled counterpart (23) or, in other form, (24), were analyzed. It was shown that the sampled AFDE (23) is asymptotically stable for certain parameter combinations while the original AFDE (22) is always unstable with infinitely many unstable poles (if $b \neq 0$ ). The transition $h \rightarrow 0$ was analyzed and it was found that the stable parameter domains of the sampled AFDE do not disappear as $h \rightarrow 0$, in spite of the fact that in the limit case when $h=0$, the system has infinitely many unstable poles. This unusual phenomenon, namely, that infinitely many unstable poles appear for an infinitesimal change in the system's parameters is not unique for FDEs, see, for example, the case of a delayed follower force in Example 3.7 in Stepan (1989).

From practical point of view, this strange transition between the sampled and the continuous cases is not very interesting, since as $h \rightarrow 0$, more and more eigenvalues get closer and closer to the unit circle. For instance, a system with many critical poles of real parts $\ln 0.99$, can be considered practically unstable, since the decay of the oscillations are extremely slow compared to the dynamics of the system.

However, the interesting point is that an advanced system with delayed feedback of the highest derivative (which is known as infinitely unstable) can be stabilized by periodic sampling of the feedback, i.e., by making the argument of the delayed term piecewise con- 
stant. This phenomenon is somewhat similar to the delayed Mathieu equation (Insperger and Stepan, 2002), where a parametric excitation in the stiffness stabilizes the delayed system. In the current case, sampling can be considered as a kind of parametric excitation in the delay as it was shown by equation (24) with (13) (see also figure 2). In this case, parametric excitation in the delay stabilizes the otherwise infinitely unstable system.

In the current analysis, one of the simplest AFDE was considered, however, similar equations may appear in many realistic problems, like in case of the stick balancing problem introduced in Section 2. In general, equations with advanced terms can typically arise in controlled mechanical systems, since the output may easily be affected by the acceleration or by the jerk of the system. This effect is often considered to be a kind of noise in the output, and is therefore often neglected, however, it may have an important role in the system's dynamics. Consider the output of a general system in the form

$$
y(t)=f\left(t, x(t-\tau), \varepsilon_{1} \dot{x}(t-\tau), \varepsilon_{2} \ddot{x}(t-\tau)\right),
$$

where $y \in \mathbb{R}^{m}$ is the output, $x \in \mathbb{R}^{n}$ is the state, and $\varepsilon_{1}$ and $\varepsilon_{2}$ are small, but nonzero parameters. Usually, such effects are neglected, i.e., $\varepsilon_{1}$ and $\varepsilon_{2}$ are considered to be zero. However, if they are not neglected, then the linearized equation has the form

$$
\dot{x}(t)=A x(t)+B_{0} x(t-\tau)+\varepsilon_{1} B_{1} \dot{x}(t-\tau)+\varepsilon_{2} B_{2} \ddot{x}(t-\tau)
$$

with $A, B_{0}, B_{1}$ and $B_{2}$ being appropriate matrices obtained during the linearization. If the parameter $\varepsilon_{2}$ is nonzero, then this equation is an AFDE, and the system is unstable with infinitely many unstable poles, independently of the exact value of $\varepsilon_{2}$. While the effect of higher order derivatives is rarely modeled in practical applications, a system's unexpected instability or its other weird behavior are often referred to as the consequence of "unmodeled system dynamics". If the system works properly then it is often attributed to the robustness of the controller and often no detailed explanation is given. In this work, it was shown that one explanation for the stable operation of such systems may be the sampling effect of digital control.

\section{Acknowledgement}

This research was supported in part by the János Bolyai Research Scholarship of the Hungarian Academy of Sciences, by the Hungarian National Science Foundation under grant no. OTKA K72911 and OTKA K68910 and by the National Science Foundation under grant DMS-0705247. The authors also acknowledge the useful discussions with Prof. John Hogan.

\section{References}

Cushing, J.M. 1977 Time delays in single species growth models, Journal of Mathematical Biology 3, 257-264.

Èl'sgol'c, L.È. 1964 Qualitative Methods in Mathematical Analysis. American Mathematical Society.

Fabiano, R.H. \& Turi, J. 1999 Preservation of stability under approximation for a neutral FDE. J. Dynamics of Continuous, Discrete and Impulsive Systems. 5, 351-364. 
Filho, O.C.O. 1997 On the asymptotic behavior of solutions of certain differential functional equations. Nonlinear analysis, Theory, Methods $\&$ Applications 30(2), 11711182.

Győri, I., Hartung, F., \& Turi, J. 1998 Preservation of stability in delay equations under delay perturbations. J. Math. Anal. Appl. 220, 290-312.

Győri I. \& Hartung, F. 2001 Preservation of stability in a linear neutral differential equation under delay perturbations. Dynamic Systems and Applications 10 225-242.

Hartung, F., Insperger, T., Stepan, G. \& Turi, J. 2006 Approximate stability charts for milling processes using semi-discretization. Applied Mathematics and Computations 174(1), 51-73.

Hale, J.K. \& Lunel, S.M.V. 1993 Introduction to Functional Differential Equations. Springer-Verlag.

Hayes, N.D. 1950 Roots of the transcendental equations associated with a certain differential-difference equations. J. London Math. Soc. 25, 226-232.

Hu, H. \& Wang, Z. 2002 Dynamics of controlled mechanical systems with delayed feedback. Springer.

Insperger, T. and Stepan, G. 2002 Stability chart for the delayed Mathieu equation. Proceedings of the Royal Society London A 458, 1989-1998.

Insperger, T., Stepan, G. \& Turi, J. 2008 On the higher-order semi-discretizations for periodic delayed systems. Journal of Sound and Vibration 313, 334-341.

Kolmanovskii, V.B. \& Myshkis, A.D. 1999 Introduction to the theory and applications of functional differential equations. Kluwer Academic Publishers.

Kolmanovskii, V.B. \& Nosov, V.R. 1986 Stability of Functional Differential equations. Academic Press.

Ladas, G. \& Stavroulakis, I.P. 1982 Oscillations caused by several retarded and advanced arguments. J. Differential Equations 44(1), 134-152.

Lv, W.J., Yang, Z.W. \& Liu, M.Z. 2007 Stability of RungeKutta methods for the alternately advanced and retarded differential equations with piecewise continuous arguments. Computers and Mathematics with Applications 54, 326-335.

Michiels, W. \& Niculescu, S.-I. 2007 Stability and stabilization of time-delay systems: an eigenvalue-based approach. Society for Industrial and Applied Mathematics.

Michiels, W., Engelborghs, K., Vansevenant, P. \& Roose, D. 2002 Continuous pole placement for delay equations. Automatica 38(5), 747-761.

Niculescu, S.-I. 2001 Delay effects on stability - A robust control approach, SpringerVerlag.

Rodrigues, I. W. 1997 Systems of differential equations of alternately retarded and advanced type. Journal of Mathematical Analysis and Applications 209, 180-190. 
Shah, S.M. \& Wiener, J. 1983 Advanced Differential Equations with Piecewise constant argument deviations. Internat. J. Math. ES Math. Sci. 6(4), 671-703.

Song, M.H., Yang, Z.W. \& Liu, M.Z. 2005 Stability of $\vartheta$-methods for advanced differential equations with piecewise continuous arguments. Computers and Mathematics with Applications 49, 1295-1301.

Stepan, G. 1989 Retarded Dynamical Systems, Longman.

Stepan, G. 2009 Delay effects in the human sensory system during balancing. Philosophical Transactions of The Royal Society A - Mathematical Physical and Engineering Sciences 367, 1195-1212.

Vyhlídal, T., Michiels, W., Zítek, P. \& McGahan, P. 2009 Stability impact of small delays in proportional-derivative state feedback. Control Engineering Practice 17, 382393.

Wiener, J. 1994 Generalized solutions of functional differential equations. World Scientific.

Winter, D.A., Patla, A.E., Prince, F., Ishac, M. \& Gielo-Perczak, K. 1998 Stiffness control of balance in quiet standing. J. Neurophys. 80, 1211-1221. 